\title{
EDITORIAL
}

\section{Contemporary issues on bone health and related diseases}

\begin{abstract}
$\Delta$ s editor of Salud Pública de México, I am pleased to $\mathrm{A}_{\text {publish this special number dedicated to analyze }}$ contemporary issues regarding the global burden represented by bone health and related diseases, primarily osteoporosis. These are acknowledged as public health problems due to the impact of their more common complication-bone fragility fractures.

I wish to thank our distinguished international editors and Mexican researchers for their collaboration. All of them are recognized scientific authorities who have made important contributions in the field of epidemiology, diagnostic methodology, pharmacological and non-pharmacological treatment, and other strategies for prevention or treatment of low bone mineral diseases such as osteoporosis and osteomalacia.

This special edition presents reviews and a comprehensive strategy useful to the daily practices of different professionals and industries related to health issues. It also contains original articles that contribute to improve clinical use of bone densitometry in Mexico, providing a robust normal reference database, as the gold standard method, to determine bone mineral density-DXA obtained from mestizos, the most common population group in Mexico.
\end{abstract}

It also provides a normal reference database for children using a peripheral DXA method. All of the above is original work reinforced by the analysis of the hereditary pattern of BMD in a cohort of three generations of Mexican women. Readers will find that the economic impact of treating osteoporotic femoral fractures with surgery through the new Mexican Health Protection System is very similar when compared with previous health strategies operating decades before.

This edition also includes two articles considered "classics" because they serve as: 1) references for treatment during the early development stages of this significant public health problem and 2) as a comprehensive review of the available pharmacologic and non-pharmacologic strategies to improve bone health and prevent bone fragility and related fractures.

As a whole, this special number of Salud Pública de Mexico makes us both proud and happy to deliver this important contribution to bone health.

Mauricio Hernández Ávila ${ }^{(1)}$ 